\title{
Autoconcepto físico, percepción de salud y satisfacción vital en una muestra de adolescentes
}

\author{
Antonio Videra-García* y Rafael Reigal-Garrido
}

Universidad de Málaga, España

\begin{abstract}
Resumen: El propósito de este trabajo es examinar las relaciones entre el autoconcepto físico, percepción de salud y satisfacción vital, en una muestra de adolescentes. Participan en el estudio 1648 adolescentes de la ciudad de Málaga (España), en edades comprendidas entre los 14 y 16 años. Los instrumentos utilizados para medir los constructos indicados son el Cuestionario de Autoconcepto Físico (CAF) de Goñi, Ruiz de Azúa y Rodríguez (2006), el Cuestionario de Salud General (GHQ) de Goldberg $(1972,1978)$ y la Escala de Satisfacción con la Vida (SWLS) elaborada por Diener, Emmons, Larsen y Griffin (1985). Los análisis de correlación y regresión lineal efectuados ponen de manifiesto la conexión entre las variables objeto de estudio, resaltando la dimensión atractivo físico y la percepción de salud como aquellas que tienen una mayor relación con la satisfacción vital, tanto en la muestra total como en función del sexo. Por otro lado, la medida complementaria de autoconcepto general, que incluye el CAF, tiene también una vinculación notable con la satisfacción vital, aunque con mayor peso en chicas que en chicos.

Palabras clave: adolescencia; autoconcepto físico; percepción de salud; satisfacción vital.
\end{abstract}

\section{Introducción}

Actualmente, el estudio del bienestar subjetivo tiene una vigencia extraordinaria, acaparando la atención de numerosos investigadores en los últimos años (Lyubomirsky y Dickerhoof, 2005; Steel, Schmidt y Shultz, 2008; Oliva et al., 2010). Concretamente, la adolescencia es un periodo muy interesante para abordar este tipo de aspectos, dado que se trata de unos años en los que la persona está en construcción y sujeta a múltiples cambios, lo que puede incidir en su desarrollo y bienestar (Kimmel y Weiner, 1998; Laca, Verdugo y Guzmán, 2005). Además, durante este tramo de la vida, la variabilidad individual otorga una dificultad más al resultado del proceso de maduración, siendo difícil predecir el resultado de la evolución en cada individuo (Rice, 2000; Mendoza, Batista y Rubio, 2005). Suponen unos años esenciales para el futuro, consolidándose la identidad individual y social, y en los que habrá que cuidar la salud, tanto física, como social y psicológica (Alvariñas, 2004; Esnaola, 2005).

Cuando se han producido intentos de analizar el bienestar subjetivo, se han diferenciado diversos factores como son su dimensión afectiva, denominada felicidad, y su componente cognitivo. A este último se le conoce como satisfacción vital, que supone un proceso de reflexión consciente mediante el cual las personas evalúan diferentes aspectos que les rodean, valorando, entre otros, si están alcanzando sus aspiraciones o si les gusta la vida que llevan (Diener, Emmons, Larsen y Griffin, 1985; Pavot y Diener, 1993; Veenhoven, 1994; García-Viniegras y González; 2000; Arita,

* Dirección para correspondencia [Correspondence address]: Antonio Videra García. Depto. Psicología Social, Antropología Social y Servicios Sociales. Facultad de Psicología. Campus de Teatinos. Universidad de Málaga. 29071, Málaga (España).E-mail: avidera@uma.es
Title: Physical self-concept, perceptions of health and life satisfaction in a sample of adolescents

Abstract: The purpose of this study is to examine the relationship between physical self-concept, perceptions of health and life satisfaction in a sample of adolescents. Participants are 1648 adolescents from the city of Malaga (Spain) aged between 14 and 16 years. The measures used to assess the constructs are the Physical Self-Concept Questionnaire (CAF; Goñi, Ruiz de Azúa \& Rodríguez, 2006), the General Health Questionnaire (GHQ; Goldberg, 1972, 1978) and the Satisfaction with Life Scale (SWLS; Diener, Emmons, Larsen \& Griffin, 1985). The correlation and linear regression analyses show relationships between the study variables, highlighting that the variables that have the strongest association with life satisfaction are the physical attractiveness dimension and perceptions of health, both in the total sample and by gender. In addition, a complementary measure of general self-concept, which includes the CAF, also has a marked relationship with life satisfaction, but this association is stronger for girls than boys.

Key words: adolescence; physical self-concept; perceptions of health; life satisfaction.

2005; Tarazona, 2005). Es difícil establecer a priori criterios que indiquen relaciones contundentes entre diversas variables y la satisfacción vital, dado que el valor otorgado a las mismas parte de criterios personales, de forma que cada individuo posee sus propios referentes (Shin y Johnson, 1978; Díaz y Sánchez-López, 2002).

En cualquier caso, diversas investigaciones han indagado en el estudio de aquellos elementos que pueden condicionar la satisfacción con la vida. En ocasiones, los investigadores se han centrado en factores externos a la persona, como el estatus socioeconómico, aunque se ha comprobado que no explican gran parte del fenómeno (Quiroga y SánchezLópez, 1997). Se ha observado, también, que el sexo es ineficaz para explicar diferencias en este tipo de percepciones (Ash y Huebner, 2001). Desde hace algunos años, se está estudiando en profundidad otras variables como los estilos de personalidad, la autonomía personal o la competencia percibida, pues se ha comprobado que pueden predecirla en un alto porcentaje de los casos (Díaz y Sánchez-López, 2001; Castro y Díaz, 2002; Nuñez, Martín-Albo y Domínguez; 2010).

Diener, Suh, Lucas y Smith (1999), en la línea de la variabilidad antes señalada, argumentan que la base de la satisfacción vital está en la percepción que tiene la persona de sí misma y del entorno en el que vive, por lo que elementos como las autopercepciones y rasgos de personalidad serán cruciales para determinarla. Las autoevaluaciones tienen un papel esencial en la percepción de la satisfacción vital, siendo el concepto que se tiene de sí mismo o la autoestima notables predictores de la misma (Furr y Funder, 1998; McCullough, Huebner y Laughlin, 2000; Ying y Fang-Biao, 2005). Podemos señalar que el autoconcepto es crucial para el desarrollo de la personalidad del individuo, estando relacionado 
con el buen funcionamiento personal y social (Goñi y Zulaica, 2000; Esnaola, Goñi y Madariaga, 2008). Hay que tener en cuenta, por otro lado, que en la adolescencia el desarrollo del autoconcepto general y específico es irregular, con fluctuaciones en función de la edad y el sexo (Esnaola, 2005).

Actualmente, se reconoce la naturaleza multidimensional del autoconcepto, siendo el modelo jerárquico de Shavelson, Hubner y Stanton (1976) uno de los más utilizados para explicar su estructura. La dimensión física es una de las más relevantes en la configuración del autoconcepto general (Fernández, Contreras, García y González, 2010), y se puede definir como las percepciones que tienen los individuos de sus habilidades y apariencia física (Stein, 1996). Tener un buen autoconcepto físico mejora la percepción de competencia personal, lo que incide en el aumento de los sentimientos de satisfacción (Alvariñas y González, 2004). Los cambios físicos en la adolescencia suponen un proceso importante, ya que generan la necesidad de adaptación a las diferentes características motoras y la aceptación de la imagen personal (Cardenal, 1999). Existen estudios, como los de Rodríguez, Goñi y Ruiz de Azúa (2006), en el que se encontraron relaciones significativas entre el autoconcepto físico y una medida de bienestar psicológico subjetivo, en una muestra de adolescentes.

Por otro lado, se puede considerar la percepción del estado de salud como otro indicador de bienestar subjetivo. De hecho, Moyano y Ramos (2007) encuentran correlaciones positivas entre la satisfacción vital y la autopercepción de salud en una muestra de chilenos de diversas edades. En diversos estudios se ha utilizado esta variable para determinar el grado de impacto que ha generado una enfermedad (Wilson y Cleary, 1995; Testa y Simonson, 1996). En este sentido, Atienza, Pons, Balaguer y García-Merita (2000) argumentan que aquellas personas que tienen una buena salud mental, careciendo, por ejemplo, de afectos negativos o ansiedad, suelen manifestar una alta satisfacción con sus vidas. La percepción de salud es un constructo al que se le otorga un valor muy importante, ya que no siempre tiene una relación directa con el estado de salud real y median otros elementos como la comparación social, los hándicaps creados a raíz de dicha situación, etc. (Arita, 2005; Argyle, 1993; Barrientos, 2005). Además, factores como la edad, el sexo o el nivel educativo se han hallado determinantes a la hora de evaluarla (Abellán, 2003).

El objetivo de este estudio ha sido observar la existencia de relaciones entre autoconcepto físico, percepción de salud y satisfacción vital en una muestra de adolescentes, así como las diferencias en función del sexo. Basados en la literatura consultada, se han buscado vínculos entre estas variables, focalizando la atención en la asociación que el autoconcepto físico y la percepción de salud tienen con la satisfacción vital. Una de las hipótesis de la investigación es que el autoconcepto físico y percepción de salud en la adolescencia tienen capacidad para predecir la evaluación que hagan los adolescentes sobre lo satisfechos que están con sus vidas. A su vez, la segunda hipótesis planteada es que el sexo genera diferen- cias en las autopercepciones objeto de estudio y en las relaciones entre ellas.

\section{Método}

Para este estudio se ha utilizado una metodología correlacional de tipo transversal, en el que se ha usado la encuesta como herramienta para la toma de datos (Salkind, 1999; Ramos, Catena y Trujillo, 2004).

\section{Participantes}

Los participantes son 1648 adolescentes de la ciudad de Málaga (España), siendo el $46.18 \%$ chicos $(n=761)$ y el $53.82 \%$ chicas $(\mathrm{n}=887)$, con edades comprendidas entre los 14 y 16 años $(M=15.26 ; D T=0.72)$. La muestra se ha obtenido del conjunto de adolescentes escolarizados que estudian los cursos de $3^{\circ}$ y $4^{\circ}$ de ESO y $1^{\circ}$ de Bachillerato en Málaga capital, siendo seleccionados mediante proceso aleatorio por conglomerados, polietápico estratificado (Ramos et al., 2004). Las unidades de primera etapa han sido los centros escolares, las de segunda etapa las aulas y las de tercera etapa los alumnos. El error para el conjunto de la muestra, utilizando la fórmula de poblaciones finitas y asumiendo la hipótesis de máxima varianza poblacional $(\mathrm{p}=\mathrm{q}=50)$ es de $2.27 \%$ al nivel de confianza del 95\%.

\section{Instrumentos}

a) Cuestionario sociodemográfico. Se han incluido varios ítems con cuestiones relativas al sexo y la edad, para situar la muestra objeto de estudio.

b) Cuestionario de Autoconcepto Físico (CAF; Goñi, Ruiz de Azúa y Rodríguez, 2006). En esta herramienta, que evalúa el autoconcepto físico, se pueden distinguir las siguientes dimensiones: habilidad física (HF), condición física (CF), atractivo físico $(\mathrm{AF})$, fuerza $(\mathrm{F})$, incluyendo además dos subescalas gracias a las que se obtienen unas medidas complementarias sobre autoconcepto físico general (AFG) y autoconcepto general (AG). Está formado por 36 ítems, 20 de ellos redactados de manera directa y 16 de forma inversa. Se contesta mediante una escala del 1 al 5, siendo 1 un grado de desacuerdo alto con lo que dicta el ítem y 5 muy de acuerdo. Los análisis de fiabilidad (Alfa de Cronbach, 1951) originales, sobre población adolescente española, fueron de .80 para habilidad física, .83 para fuerza, 84 para condición física y .88 para atractivo físi$\mathrm{co}$, para las subescalas de autoconcepto físico general fue de .88 y para la de autoconcepto general .79 .

c) Cuestionario de Salud General (GHQ-30; General Health Questionnaire - Goldberg, 1972, 1978; Muñoz, VázquezBarquero, Rodríguez, Pastrana y Varo; 1979). Este cuestionario fue diseñado inicialmente para detectar trastornos psíquicos en un ámbito comunitario y en medios clínicos no psiquiátricos, y se centra en los componentes psicológicos que identifican una salud negativa (Gold- 
berg y Williams, 1996). Nosotros hemos utilizado una versión corta de 30 ítems, aunque existen otras como las de 12, 28, 36, 60 o 140 (Godoy-Izquierdo, Godoy, López-Torrecillas y Sánchez-Barrera, 2002). Hay cuatro posibles respuestas para cada ítem y, como indica Veiga (2004), los modos de respuesta pueden ser varios: puntuación GHQ (0-0-1-1), puntuación Likert simple (0-1-23) y puntuación Likert modificada (0-0-1-2). En este estudio se ha utilizado la puntuación Likert simple, en la que 0 significa ausencia de problemas, es decir, buena salud, y 3 indica la presencia de problemas de salud. El GHQ-30 ha sido utilizado en diversos estudios, como el realizado por Lobo y Gimeno (1981) en población española con valores de consistencia interna del mismo de .91 (Alfa de Cronbach).

d) Escala de Satisfacción Vital (SWLS; Satisfaction With Life Scale - Diener et al., 1985). Este instrumento surgió con la idea de medir la satisfacción con la vida obteniendo un valor general sobre dicho constructo. En el estudio original, realizado sobre población universitaria, el instrumento alcanzó una buena consistencia interna $(\alpha=.87)$ y un coeficiente de correlación test-retest alto $(r=.82)$. Ha sido utilizado en otros estudios, como los de Martínez, Buelga y Cava (2007) sobre problación adolescente, en el que mostró también un adecuado índice de fiabilidad $(\alpha=.74)$. Los participantes del estudio utilizaron para responder una escala tipo Likert con 7 posibles respuestas, desde muy en desacuerdo (puntuación= 1) hasta muy de acuerdo (puntuación= 7) con lo que indica cada ítem. Esta escala tiene su correspondiente adaptación y validación al castellano gracias a Atienza et al. (2000), precisamente en población adolescente.

\section{Procedimiento}

Para la recogida de datos se ha ido a centros escolares, pidiendo permiso previamente para que los alumnos pudieran participar en el estudio. Posteriormente, se solicitó el consentimiento por escrito de los padres para que pudieran formar parte de la investigación, explicando a los adolescentes que sería una participación anónima y voluntaria. Los cuestionarios han sido auto-administrados, aunque explicándolo adecuadamente y estando presente mientras eran cumplimentados para resolver posibles dudas, controlando así que se completaban adecuadamente. Se rellenaron en el aula, siendo la duración media de 30 minutos. Inicialmente, el número de adolescentes que accedieron a colaborar fue de 1765, pero tras desechar algunos cuestionarios por no estar debidamente cumplimentados, la muestra ha quedado compuesta finalmente por 1648 .

\section{Análisis de los datos}

Se han realizado en este trabajo análisis descriptivos e inferenciales. Para evaluar las diferencias en función del sexo en autoconcepto físico, percepción de salud y satisfacción vi- tal se ha utilizado un análisis de medias t-student. Para comprobar la existencia de relaciones entre el autoconcepto, la percepción de salud y satisfación vital, se ha empleado un análisis de correlaciones (Pearson) y de regresión lineal múltiple. Para el tratamiento informático se ha usado el paquete estadístico SPSS en su versión 15.0.

\section{Resultados}

\section{Análisis descriptivo y fiabilidad de los instrumentos}

En la siguiente tabla se puede observar la distribución de los datos, así como la fiabilidad de los mismos.

Tabla 1. Estadísticos descriptivos y análisis de la fiabilidad de los instrumentos.

\begin{tabular}{lccccc}
\hline & Media & DT & Asimetría & Curtosis & $\begin{array}{c}\text { Alfa de } \\
\text { Cronbach }\end{array}$ \\
\hline Habilidad física & 3.48 & .85 & -.46 & -.32 & .77 \\
Condición física & 3.29 & .95 & -.20 & -.71 & .83 \\
Atractivo físico & 3.39 & .90 & -.43 & -.54 & .79 \\
Fuerza & 3.05 & .86 & .10 & -.51 & .78 \\
A. F. General & 3.65 & .89 & -.67 & -.09 & .81 \\
A. General & 3.96 & .74 & -.76 & .16 & .73 \\
Percepción & 1.00 & .42 & .65 & .59 & .91 \\
de salud & & & & & .10 \\
Satisfacción vital & 4.93 & 1.21 & -.34 & .83 \\
\hline
\end{tabular}

Como se puede observar, los valores de asimetría y curtosis cumplen con el criterio de normalidad propuesto por Curran, West and Finch (1996). Los análisis de fiabilidad realizados (Alfa de Cronbach) muestran unos niveles de consistencia interna adecuados, que oscilan entre los valores .73 y .91 (Tabla 1$)$.

\section{Análisis de CAF, GHQ y SWLS en función del sexo}

La tabla mostrada a continuación refleja las diferencias entre chicos y chicas en las medidas de los cuestionarios utilizados.

Tabla 2. Estadísticos descriptivos y análisis de las diferencias en función del sexo.

\begin{tabular}{lllllll}
\hline & $\begin{array}{l}\text { Media } \\
\text { Chicos }\end{array}$ & $D T$ & $\begin{array}{l}\text { Media } \\
\text { chicas }\end{array}$ & DT & $t$ & \multicolumn{2}{l}{$t$} \\
\hline Habilidad física & 3.73 & .77 & 3.27 & .86 & 11.55 & .000 \\
Condición física & 3.67 & .87 & 2.97 & .90 & 15.80 & .000 \\
Atractivo físico & 3.53 & .82 & 3.28 & .96 & 5.72 & .000 \\
Fuerza & 3.43 & .81 & 2.73 & .78 & 17.32 & .000 \\
A. F. General & 3.83 & .81 & 3.49 & .92 & 7.83 & .000 \\
A. General & 4.00 & .74 & 3.93 & .74 & 2.07 & .038 \\
Percepción & .94 & .41 & 1.06 & .43 & -5.46 & .000 \\
de salud & & 1.16 & 4.89 & 1.25 & 1.53 & .126 \\
Satisfacción vital & 4.98 & 1.53 &
\end{tabular}

Los análisis realizados indican que los chicos tienen mejor percepción que las chicas en habilidad física $\left(t_{1643.28}=\right.$ $11.55 ; p<.001)$, condición física $\left(t_{1646}=15.80 ; p<.001\right)$, atractivo fisico $\left(t_{1645.97}=5.72 ; p<.001\right)$ y fuerza $\left(t_{1646}=17.32 ; p<.001\right)$, 
siendo en todos los casos estadísticamente significativo. Además, las escalas complementarias del CAF ofrecen datos que reflejan una mejor valoración del autoconcepto físico general $\left(t_{1644.89}=7.83 ; p<.001\right)$ y del autoconcepto general $\left(t_{1646}=2.07\right.$; $p<.05)$ por parte de ellos (Tabla 2$)$. En cuanto a la percepción de salud $\left(t_{1634.30}=-5.46 ; p<.001\right)$, las chicas obtienen mayor puntuación, lo que indica la presencia de mayores problemas de salud que los chicos, siendo también las diferencias significativas. Respecto a la satisfacción vital $\left(t_{1636.06}=1.53\right.$; $p>$.05), los resultados señalan una puntuación similar, sin diferencias significativas entre los dos grupos (Tabla 2).

Para comprobar las relaciones existentes entre las variables del estudio, se ha realizado un análisis de correlación (Pearson), tal y como podemos ver en la Tabla 3.

Tabla 3. Análisis de correlaciones (Pearson).

\begin{tabular}{|c|c|c|c|}
\hline & $\begin{array}{l}\text { SWLS } \\
\text { Total }\end{array}$ & $\begin{array}{l}\text { SWLS } \\
\text { chicos }\end{array}$ & $\begin{array}{l}\text { SWLS } \\
\text { chicas }\end{array}$ \\
\hline Habilidad física & $.18^{* *}$ & $.16^{* *}$ & $.19^{* *}$ \\
\hline Condición física & $.21 * *$ & $.20^{* *}$ & $.22 * *$ \\
\hline Atractivo físico & $.36 * *$ & $.31 * *$ & $.39 * *$ \\
\hline Fuerza & $.16^{* *}$ & $.23^{* *}$ & $.10^{* *}$ \\
\hline A. físico general & $.34 * *$ & $.30 * *$ & $.37 * *$ \\
\hline A. general & $.43^{* *}$ & $.34 * *$ & $.50 * *$ \\
\hline Percepción de salud & $-.50 * *$ & $-.46 * *$ & $-.53 * *$ \\
\hline
\end{tabular}

En función de los resultados obtenidos, se puede ver que las diferentes subescalas del CAF y los valores del GHQ correlacionan significativamente con los de SWLS. Sin embargo, cabe distinguir varios grupos. En primer lugar, hay una correlación baja entre habilidad física y satisfacción vital, tanto para la muestra total $\left(r_{1648}=.18 ; p<.01\right)$ como para chicos $(r$ $\left.{ }_{1648}=.16 ; p<.01\right)$ y chicas $\left(r_{1648}=.19 ; p<.01\right)$. También ocurre con condición física $\left(r_{1648}=.21 ; p<.01\right)$ y fuerza $\left(r_{1648}=.16\right.$; $p<.01)$ respecto a la satisfacción vital, tanto para la muestra total como por sexo.

En segundo lugar, hay que señalar un grupo en el que las correlaciones son más elevadas, con un valor moderado según Cohen (1998), como las establecidas entre atractivo físico y satisfacción vital, tanto para la muestra total $\left(r_{1648}=.36 ; p<\right.$ $.01)$ como por sexo (chicos, $r_{1648}=.31 ; p<.01$ y chicas, $r$ $\left.{ }_{1648}=.39 ; p<.01\right)$, o entre autoconcepto físico general y satisfacción vital (muestra total; $\mathrm{r}_{1648}=.34 ; p<.01$; chicos, $\mathrm{r}_{1648}=.30 ; p<$ .01 y chicas, $r_{1648}=.37 ; p<.01$ ). Las correlaciones más altas se establecen entre satisfacción vital con el autoconcepto general $(r$ $\left.{ }_{1648}=.43 ; p<.01\right)$ y percepción de salud $\left(r_{1648}=-.50 ; p<.01\right)$. En chicos, el autoconcepto general tiene una correlación más baja $(r$ $\left.{ }_{1648}=.34 ; p<.01\right)$ que en las chicas $\left(r_{1648}=-.50 ; p<.01\right)$, aunque la percepción de salud correlaciona de una forma importante tanto en chicos $\left(r_{1648}=-.46 ; p<.01\right)$ como en chicas $(r$ $\left.{ }_{1648}=-.53 ; p<.01\right)$.

Además de observar las correlaciones entre las diferentes variables, y siguiendo el planteamiento desarrollado en la introducción, se ha analizado la capacidad predictiva que las variables presentes en el cuestionario de autoconcepto físico y percepción de salud tienen sobre la satisfacción vital. Se ha

empleado un análisis de regresión lineal usando el método de pasos sucesivos. Como se puede ver en la Tabla 4, el resumen del modelo indica que se han incluido las variables percepción de salud, atractivo físico y autoconcepto general, tanto para la muestra total como para las chicas, aunque el realizado para los chicos únicamente lo hace con percepción de salud y atractivo físico.

Tabla 4. Análisis de regresión lineal (pasos sucesivos) aplicados sobre la variable satisfacción vital.

\begin{tabular}{lllllll}
\hline Variable criterio & $\begin{array}{l}\text { Variables } \\
\text { predictoras }\end{array}$ & $\mathrm{R}^{2}$ & $\begin{array}{l}\mathrm{R}^{2} \\
\text { corregida }\end{array}$ & Beta & $\mathrm{T}$ \\
\hline SWLS & & .55 & .30 & .30 & & \\
(Muestra total) & $\mathrm{GHQ}$ & & & & -.368 & $-15.48^{* * *}$ \\
& $\mathrm{AG}$ & & & & .165 & $6.07^{* * *}$ \\
& $\mathrm{AF}$ & & & & .140 & $5.56^{* * *}$
\end{tabular}

SWLS (Chicos)

SWLS

(Chicas)

AF

$* p<.05 ; * * p<.01 ; * * * p<.001$

Para la muestra total, los análisis indican que las variables explican el $30 \%$ de la varianza $\left(\mathrm{R}=.55 ; \mathrm{R}^{2}\right.$ corregida $=.30$; $\mathrm{F}=239.99 ; p<.001)$, con un mayor peso de la percepción de salud (beta $=-.368 ; p<.001$ ), seguido del autoconcepto general (be$\left.t_{a}=.165 ; p<.001\right)$ y el atractivo físico (beta $\left.=.140 ; p<.001\right)$. En chicos, el modelo explica el $25 \%\left(\mathrm{R}=.50 ; \mathrm{R}^{2}\right.$ corregida $=.25$; $\mathrm{F}=125.50 ; p<.001)$, con una mayor importancia de la percepción de salud (beta $=-.402 ; p<.001$ ) que el atractivo físico (beta= .208 ; $p<.001)$. Por último, para las chicas, el análisis de regresión explica el $35 \%$ de la varianza $\left(\mathrm{R}=.60 ; \mathrm{R}^{2}\right.$ corregida $=$ $.35 ; \mathrm{F}=163.15 ; p<.001)$, con unos valores más altos para la percepción de salud (beta $=-.361 ; p<.001)$, seguido del autoconcepto general (beta $=.256 ; p<.001)$ y el atractivo físico (beta $=.097$; $p<.01)$.

\section{Discusión}

Los resultados indican que los chicos tienen mejor percepción de autoconcepto físico que las chicas en todas las subescalas del cuestionario CAF, incluidas las medidas complementarias referidas al autoconcepto físico general y autoconcepto general. Estos datos están en consonancia con los hallados en otros estudios (Fox y Corbin, 1989; Goñi, Ruiz de Azúa y Liberal, 2004; Goñi, Ruiz de Azúa y Rodríguez, 2004; Hayes, Crocker y Kowalski, 1999; Klomsten, Skaalvik y Espens, 2004; Moreno y Cervelló, 2005). Aunque hay investigaciones que no encuentran diferencias en algunos de los factores del cuestionario, como en atractivo físico y autoconcepto general (Molero, Ortega, Valiente y Zagalaz, 2010), la mayoría de los diversos investigadores encuentran a las chicas más preocupadas por su aspecto físico, lo que in- 
cide en una mayor exigencia y menor satisfacción corporal, ocurriendo en una amplia franja de edad (Collins, 1991; Cruz y Maganto, 2002).

En relación a la percepción de salud, el trabajo pone de manifiesto diferencias significativas en función del sexo, teniendo los chicos una mejor valoración de la misma. Diversos estudios han señalado que el sexo es una variable que discrimina la percepción de salud entre las personas, siendo más favorable en hombres que en mujeres y haciéndose patente en todas las edades, aunque el fenómeno sea más evidente cuando se alcanza una edad madura (Abellán, 2003; García, Matute, Tifner, Gallizo y Gil-Lacruz, 2007). Los resultados analizados respecto a la satisfacción vital permiten observar que no hay diferencias significativas entre los adolescentes en función del sexo, lo que está en línea con lo encontrado en otros trabajos, realizados tanto en edad adolescente como en adultos (Ash y Huebner, 2001; Huebner, Drane y Valois, 2000; Zegers, Rojas-Barahona y Förster, 2009).

Los análisis de correlación y regresión lineal efectuados indican que existen relaciones significativas entre las variables objeto de estudio. En concreto, se han reproducido aquellos vínculos que la literatura consultada planteaba entre las variables autoconcepto y percepción de salud con la satisfacción vital. Diversos autores argumentan que lo satisfechos que estamos con nuestra vida se trata de de un juicio consciente mediante el cual valoramos diferentes aspectos, como los objetivos alcanzados en función de nuestras expectativas, el estado de nuestra salud, la propia imagen corporal, la red social y el apoyo que nos brinda, etc. (Pavot, Diener, Colvin y Sandvik, 1991; Pavot y Diener, 1993; Wilson y Cleary, 1995; Rodríguez et al., 2006). Además, estas evaluaciones son el resultado de una forma particular de percibir el contexto que nos rodea, por lo que este constructo está fuertemente condicionado por la interpretación que se haga de las situaciones vividas y personales (Diener, et al., 1999).

Por lo tanto, tiene sentido plantear la influencia de este tipo de autopercepciones, sobre todo en una etapa tan delicada de la vida como es la adolescencia. En este periodo acontecen numerosos cambios, a los que hay que adaptarse, como los que suceden en la imagen corporal, y que pueden provocar alteraciones en el bienestar (Carraro, Scarpa y Ventura, 2010). En este estudio, se ha podido observar que las diferentes dimensiones del autoconcepto físico correlacionan significativamente con la percepción de satisfacción vital. En este sentido, se ha comprobado también que las chicas obtienen una mayor correlación, que los chicos, entre prácticamente todas las subescalas del autoconcepto físico y la satisfacción vital, salvo en fuerza. Estos resultados reflejan la importancia de las percepciones físicas en esta franja de edad adolescente, ejerciendo un peso en las chicas bastante significativo, destacando el atractivo físico por encima de las demás dimensiones del autoconcepto físico. De hecho, en los análisis de regresión lineal el atractivo físico emerge como una variable con una importante capacidad predictiva de la satisfacción vital, lo que contribuye a corroborar la importancia de este factor (Franzoi y Shields, 1984; Koff, Rierdan y Stubbs, 1990; Goñi e Infante, 2010).

Una de las subescalas del instrumento utilizado pone de manifiesto la importancia del autoconcepto general. Tanto en la muestra general como por sexos, las correlaciones entre esta variable y la satisfacción vital han sido evidentes, aunque en mayor medida en chicas. De hecho, el modelo de regresión propuesto otorga valores significativos a este factor sobre la predicción de la satisfacción vital, lo que no ocurre en chicos. En cualquier caso, se hace patente la importancia del mismo, tal o como manifestaban Taylor y Fox (2005), argumentando que las personas que tienen un mayor nivel de autoconcepto manifiestan de forma más positiva lo satisfechas que están con sus vidas. Además, autores como Neugarten, Havighurst y Tobin (1996) le otorgan un valor muy alto a este hecho, considerando que el autoconcepto que tengamos está en la base de lo satisfechos que estemos con nuestra vida.

De igual forma, los datos indican la relación existente entre percepción de salud y satisfacción vital. Tanto el análisis de correlaciones como el modelo de regresión propuesto así lo manifiestan, además con unos índices de significación considerables y tanto para la muestra total como en función del sexo. Autores como Atienza et al. (2000) ponen de manifiesto la importancia de la salud y ausencia de factores como la depresión o ansiedad en la respuesta positiva de las personas ante la valoración sobre lo satisfechas que estaban con sus vidas. Otros investigadores en este campo señalan que la valoración personal de nuestro estado de salud es realmente lo importante, dado que son juicios que repercuten, en gran medida, sobre la forma de actuar, pensar y sentir (Shin y Johnson, 1978; Diener et al., 1985; Díaz y Sánchez-López, 2002). Castro y Sánchez (2000) consideran que cuando en las personas ocurre un fenómeno en el que se asocia una mejor autopercepción de salud con mayores logros y éxitos pasados y futuros, lo que refleja uno de los aspectos más relevantes en el estudio de la satisfacción vital como es el cumplimiento de los objetivos marcados y el alcance de las expectativas en la vida.

Se puede concluir diciendo que se han hallado datos que indican la relevancia que tiene el autoconcepto físico, así como la valoración general del concepto personal, y la percepción de salud sobre la satisfacción con la vida. En concreto, en un periodo de la adolescencia como el estudiado en este trabajo, tan crítico para el desarrollo de los individuos, y que corrobora las teorías que señalan a los factores internos como determinantes claves para una evaluación positiva del bienestar. 


\section{Referencias}

Abellán, A. (2003). Percepción del estado de salud. Revista Multidisciplinar de Gereontología, 13(5), 340-342.

Alvariñas, M. (2004). Atribución casual en educación física y estilo de vida saludable. Tesis doctoral. Universidad de A Coruña.

Alvariñas, M. y González, M. (2004). Relación entre la práctica físicodeportiva extraescolar y el autoconcepto físico en la adolescencia. Revista de Educación Física, 94, 5-8.

Argyle, M. (1993). Psicología y calidad de vida. Intervención psicosocial, 2(6), 515.

Arita, B. (2005). Satisfacción por la vida y teoría homeostática del bienestar. Psicología y Salud, 15(1), 121-126.

Ash, C. y Huebner, E.S. (2001). Environmental events and life satisfaction reports of adolescents: A Test of Cognitive Mediation. School Psychology International, 22(3), 320-336.

Atienza, F.L., Pons, D., Balaguer, I. y García-Merita, M. (2000). Propiedades psicométricas de la Escala de Satisfacción con la Vida en adolescentes. Psicothema, 12(2), 314-319.

Barrientos, J. (2005). Calidad de vida. Bienestar subjetivo, una mirada psicosocial. Santiago: Universidad Diego Portales.

Cardenal, V. (1999). El autoconcepto y la autoestima en el desarrollo de la madurez personal. Málaga: Aljibe.

Carraro, A., Scarpa, S. y Ventura, L. (2010). Relationships between physical self-concept and physical fitness in italian adolescents. Perceptual and Motor Skills, 110(2), 522-530.

Castro, A. y Díaz, J.F. (2002). Objetivos de vida y satisfacción vital en adolescentes españoles y argentinos. Psicothema, 14(1), 112-117.

Castro, A. y Sánchez, P. (2000). Objetivos de vida y satisfacción autopercibida en estudiantes universitarios. Psicothema, 12(1), 87-91.

Cohen, J. (1998). Statistical power analysis for the behavioural sciences $\left(2^{a}\right.$ ed.). Hillsdale, N.J.: Erlbaum.

Collins, M.E. (1991). Body figure perceptions and preferences among preadolescent children. International Journal of Eating Disorders, 10(2), 199208.

Cronbach, L.J. (1951). Coefficient alpha and the internal structure of test. Psychometrika, 16, 297-234.

Cruz, S. y Maganto, C. (2002). Índice de masa corporal, preocupación por la delgadez y satisfacción corporal en adolescentes. Revista de Psicología General y Aplicada, 55(3), 455-473.

Curran, P.J., West, S.G. y Finch, J.F. (1996). The robustness of the Test Statistics to Nonnormality and Specification Error in Confirmatory Factor Analysis. Psychological Methods, 1(1), 16-29.

Díaz, J.F. y Sánchez-López, M.P. (2001). Relevancia de los estilos de personalidad y las metas personales en la predicción de la satisfacción vital. Anales de Psicología, 17(2), 151-158.

Díaz, J.F. y Sánchez-López, M.P. (2002). Relaciones entre estilos de personalidad y satisfacción autopercibidas en diferentes áreas vitales. Psicothema, 14(1), 100-105.

Diener, E., Emmons, R., Larsen, R.J. y Griffin, S. (1985). The satisfaction with life scale. Journal of Personality Assessment, 49(1), 71-75.

Diener, E., Suh, E., Lucas, R. y Smith, H. (1999). Subjetive well-being: three decades of progress. Psychological Bulletin, 125(2), 276-302.

Esnaola, I. (2005). Desarrollo del autoconcepto durante la adolescencia y principio de la juventud. Revista de Psicología General y Aplicada, 58(2), 265-277.

Esnaola, I., Goñi, A. y Madariaga, J.M. (2008). El autoconcepto: perspectivas de investigación. Revista de Psicodidáctica, 13(1), 179-194.

Fernández, J.G., Contreras, O.R., García, L.M. y González, S. (2010). Autoconcepto físico según la actividad físicodeportiva realizada y la motivación hacia ésta. Revista Latinoamericana de Psicología, 42(2), 251-263.

Fox, K.R. y Corbin, C.B. (1989). The physical self-perception profile: development and preliminary validation. Journal of Sport and Exercise Psychology, 11(4), 408-430.

Franzoi, S.L. y Shields, S.A. (1984). The Body Steem Scale: Multidimensional structure and sex differences in a college population. Journal of Personality Assessment, 48(2), 173-178.

Furr, R.M. y Funder, D. (1998). A multimodal analysis of personal negativity. Journal of personality and social psychology, 74(6), 1580-1591.
García, Y., Matute, S., Tifner, S., Gallizo, M.E. y Gil-Lacruz, M. (2007). Sedentarismo y percepción de la salud: Diferencias de género en una muestra aragonesa. Revista Internacional de Medicina y Ciencias de la Actividad Física y el Deporte, 7(28), 344-358.

García-Viniegras, C. y González, I. (2000). La categoría bienestar psicológico, su relación con otras categorías sociales. Revista Cubana de Medicina Integral, 16(6), 586-592.

Godoy-Izquierdo, D., Godoy, J.F., López-Torrecillas, F. y Sánchez-Barrera M.B. (2002). Propiedades psicométricas de la versión española del "Cuestionario de Salud General de Goldberg-28". Revista de Psicología de la Salud, 14(1), 49-71.

Goldberg, D.P. (1972). The detection of psychiatric illness by questionnaire. London: Oxford University Press.

Goldberg, D.P. (1978). Manual of the General Health Questionnaire. Windsor, Inglaterra: NLF Publishing.

Goldberg, D.P. y Williams, P. (1996). Cuestionario de Salud General GHQ. Barcelona: Masson.

Goñi, A., Ruiz de Azúa, S. y Liberal, I. (2004). Propiedades psicométricas de un nuevo cuestionario para la medida del autoconcepto físico. Revista de Psicología del Deporte, 13(2), 195-213.

Goñi, A., Ruiz de Azúa, S. y Rodríguez, A. (2004). Deporte y autoconcepto físico en la preadolescencia. Apunts, 77, 18-24.

Goñi, A., Ruiz de Azúa, S. y Rodríguez, A. (2006). Cuestionario de Autoconcepto Fïsico (CAF). Manual. Madrid: EOS.

Goñi, A. y Zulaica, L.M. (2000). La participación en el deporte escolar y el autoconcepto en escolares de 10 a 11 años de la provincia de Guipúzcoa. Apunts, 59, 6-10.

Goñi, E. e Infante, G. (2010). Actividad físico-deportiva, autoconcepto físico y satisfacción con la vida. European Journal of Education and Psychology, 3(2), 199-208.

Hayes, S.D., Crocker, P.R.E. y Kowalski, K.C. (1999). Gender differences in physical self-perceptions, global self-esteem, and physical activity: evaluation of the Physical Self-Perception Profile model. Journal of Sport Behaviour, 22(1), 1-14.

Huebner, E.S., Drane, J.W., y Valois R. F. (2000). Levels and demographic correlates of adolescent life satisfaction reports. School Psychology International, 21(3), 281- 292.

Kimmel, D.C. y Weiner, I.B. (1998). La adolescencia: una transición del desarrollo. Barcelona: Ariel Psicología.

Koff, E., Rierdan, J. y Stubbs, M.L. (1990). Gender, body image, and selfconcept in early adolescence. Journal of Early Adolescence, 10(1), 56-68.

Klomsten, A.T., Skaalvik, E.M. y Espens, G.A. (2004). Physical self-concept and sports: do gender differences still exist?. Sex Roles, 50(1/2), 119-127.

Laca, F.A., Verdugo, J.C. y Guzmán, J. (2005). Satisfacción con la vida de algunos colectivos mexicanos: una discusión sobre la psicología del bienestar subjetivo. Revista de Enseñanza e Investigación en Psicología, 10(2), 325 336.

Lobo, A. y Gimeno, J.L. (1981). Estudio transcultural de los problemas psiquiátricos en pacientes oncológicos. Oncología 80, 136, 25-30.

Lyubomirsky, S., y Dickerhoof, R. (2005). Subjective well-being. En J. Worrell (Ed.), Handbook of girls' and women's psychological health: Gender and wellbeing across the life span (pp. 166-174). New York: Oxford University Press.

Martínez, M., Buelga, S. y Cava, M.J. (2007). La satisfacción con la vida en la adolescencia y su relación con la autoestima y el ajuste escolar. Anuario de Psicología, 38(2), 5-15.

McCullough, G., Huebner, E.S. y Laughlin, J.E. (2000). Life events, self concept and adolescents' positive subjective well-being. Psychology in the Schools, 37(3), 281-290.

Mendoza, R., Batista, J.M. y Rubio, A. (2005). El desarrollo de estilos de vida en los adolescentes escolarizados: diferencias entre chicos y chicas. Revista de Psicología General y Aplicada, 58(1), 51-74.

Molero, D., Ortega, F., Valiente, I. y Zagalaz, M.L. (2010). Estudio comparativo del autoconcepto físico en adolescentes en función del género y del nivel de actividad físico-deportiva. Retos, 17, 38-41.

Moreno, J.A. y Cervelló, E. (2005). Physical self-perception in spanish adolescents: effects of gender and involvent in physical activity. Journal of Human Movement Studies, 48, 291-311. 
Moyano, E. y Ramos, N. (2007). Bienestar subjetivo: midiendo satisfacción vital, felicidad y salud en población chilena de la Región Maule. Universum: Revista de Humanidades y Ciencias Sociales, 2(22), 185-200.

Muñoz, P.E., Vázquez-Barquero, J.L., Rodríguez, F., Pastrana, E. y Varo, J. (1979). Adaptación española del General Health Questionnaire (GHQ) de D.P. Goldberg. Archivos de Neurobiologia, 42(2), 139-158.

Neugarten, B.L., Havighurst, R.J. y Tobin, S.S. (1996). The measurement of Life Satisfaction. En D.A. Neugarten (Ed.), The meanings of age. Selected papers of Bernice L. Neugarten (pp. 296-323). Chicago: The University of Chicago Press.

Nuñez, J.L., Martín-Albo, J. y Domínguez, E. (2010). Propiedades psicométricas de la Escala de Satisfacción con la Vida en sujetos practicantes de actividad física. Revista de Psicología del Deporte, 19(2), 291-304.

Oliva, A., Ríos, M., Antolín, L., Parra, A., Hernando, A. y Pertegal, A. (2010). Más allá del déficit: Construyendo un modelo de desarrollo positivo adolescente. Infancia y Aprendizaje, 33(2), 223-234.

Pavot, W. y Diener, E. (1993). Review of the Satisfaction with Life Scale. Psychological Assesment, 5(2), 164-172.

Pavot, W., Diener, E.D., Colvin, C.R. y Sandvik, E. (1991). Further validation of the Satisfaction With Life Scale: Evidence for the cross-met-hod convergence of well-being. Journal of Personality Assessment, 57(1), 149161.

Quiroga, Mª.A. y Sánchez, Mª.P. (1997). Análisis de la insatisfacción familiar. Psicothema, 9(1), 69-82

Ramos, M.M., Catena, A. y Trujillo, H.M. (2004) Manual de métodos y técnicas de investigación en ciencias del comportamiento. Madrid: Biblioteca Nueva.

Rice, F.P. (2000). Adolescencia. Desarrollo, relaciones y cultura. Madrid: Prentice Hall.

Rodríguez, A., Goñi, A. y Ruiz de Azúa (2006). Autoconcepto físico y estilos de vida en la adolescencia. Intervención Psicosocial, 15(1), 81-94.

Salkind, N.J. (1999). Métodos de investigación. México: Prentice Hall.

Shavelson, R.J., Hubner, J.J. y Stanton, J.C. (1976). Self-concept: validation of construct interpretations. Review of Educational Research, 46(3), 407441.

Shin, D.C. y Johnson, D.M. (1978). Avowed happiness as an overall assessment of the quality of life. Social Indicators Research, 5(1/4), 474-492.
Steel, P., Schmidt, J. y Shultz, J. (2008). Refining the relationship between personality and subjective well-being. Psychological bulletin, 134(1), 138161

Stein, , R.J. (1996). Physical self-concept. En B.A. Braken (Ed.), Handbook of self-concept. Developmental, social and clinical consideration (pp. 374-394). New York: Wiley.

Tarazona, D. (2005) Autoestima, satisfacción con la vida y condiciones de habitabilidad en adolescentes estudiantes de quinto año de media. Un estudio factorial según pobreza y sexo. Revista de Investigación en Psicología, $8(2), 57-65$.

Taylor, A. y Fox, K. (2005). Effectiveness of a primary care exercise referral intervention for changing physical self-perceptions over nine months. Health Psychology, 24(1), 11-21.

Testa, M.A. y Simonson, D.C. (1996). Assesment of quality of life outcomes. New England Journal of Medicine, 334(13), 835-840.

Veenhoven, R. (1994). El estudio de la satisfacción con la vida. Intervención Psicosocial, 3(9), 87-116.

Veiga, O.L. (2004). Género, refuerzo social y actitud hacia el deporte como determinantes de la práctica de actividad física y deportiva en el tiempo de ocio de escolares adolescentes. Análisis de sus relaciones con el estado de salud percibida. Estudio sobre la población del municipio madrileño de Leganés. Tesis doctoral. Universidad Politécnica de Madrid.

Wilson, I.B. y Cleary, P.D. (1995). Linking clinical variables with HealthRelated Quality of Life. A conceptual model of patient outcomes. Journal of the American Medical Association, 273(1), 59-65.

Ying, S. y Fang-Biao, T. (2005). Correlations of school Life Satisfaction, Self-Esteem and Coping Style in Middle School Students. Chinese Mental Health Journal, 19(11), 741-744.

Zegers, B., Rojas-Barahona, C. y Förster, C. (2009). Validez y confiabilidad del Índice de Satisfacción Vital (LSI-A) de Neugarten, Havighurst y Tobin en una Muestra de adultos y adultos mayores en Chile. Terapia psicológica, 27(1), 15-26.

(Articulo recibido: 05-07-2011, revisión: 02-02-2012, aceptado: 3-02-2012) 